\title{
Erratum to: Immunoanalysis for environmental monitoring and human health
}

\author{
Shirley J. Gee ${ }^{1}$ - Ivan R. Kennedy ${ }^{2} \cdot$ N. Alice Lee ${ }^{3} \cdot$ Hideo Ohkawa ${ }^{4}$. \\ Tippawan Prapamontol ${ }^{5} \cdot \mathrm{Ting}_{\mathrm{Xu}^{6}}$
}

Received: 30 June 2016 / Accepted: 30 June 2016 / Published online: 13 July 2016

(C) Springer-Verlag Berlin Heidelberg 2016

\section{Erratum to: Anal Bioanal Chem}

DOI:10.1007/s00216-016-9666-X

The original version of this article unfortunately contained a mistake. The name and affiliation of the third author were incorrect. The correct version is presented in this paper and the original article was corrected.

Published in the topical collection Immunoanalysis for Environmental Monitoring and Human Health with guest editors Shirley J. Gee, Ivan R. Kennedy, N. Alice Lee, Hideo Ohkawa, Tippawan Prapamontol, and Ting Xu.

The online version of the original article can be found at http://dx.doi.org/ 10.1007/s00216-016-9666-x.

Shirley J. Gee

sjgee@ucdavis.edu

Ivan R. Kennedy

ivan.kennedy@sydney.edu.au

N. Alice Lee

alice.lee@unsw.edu.au

Tippawan Prapamontol

tippawan.prapamontol@cmu.ac.th

Ting Xu

xuting@cau.edu.cn

1 Department of Entomology and Nematology, University of California, One Shields Avenue, Davis, CA 95616, USA
2 Faculty of Agriculture and Environment, University of Sydney, Sydney, NSW 2006, Australia

3 ARC Training Centre for Advanced Technologies in Food Manufacture, School of Chemical Engineering, University of New South Wales, Sydney 2052, Australia

4 Kobe University, Kashiodai 14-14, Kitaku, Kobe 651-1255, Japan

5 Research Institute for Health Sciences, Chiang Mai University, P.O. Box 80 C.M.U., Chiang Mai 50202, Thailand

6 College of Resources and Environmental Sciences, China Agricultural University, No. 2 Yuan Ming Yuan West Road, Haidian District, Beijing 100193, China 